\title{
COORDENAÇÃO DA INDÚSTRIA DE COURO BRASILEIRO: ABORDAGEM BASEADA NO COEFICIENTE DE VARIAÇÃO
}

\author{
Coordination of the Brazilian Leather Industry: Approach Based on Coefficient of Variation
}

\begin{abstract}
RESUMO
Neste estudo objetivou-se caracterizar o desempenho da coordenação na indústria de couro, por meio da medição da variabilidade da quantidade de couro recebido e adquirido para curtimento entre os anos de 1999 a 2012. Para isso, utiliza a Teoria dos Custos de transação como estrutura analítica para discutir a coordenação e a estrutura de governança da indústria do couro. Os dados foram extraídos dos censos trimestrais realizados pelo Instituto Brasileiro de Geografia e Estatística (IBGE). Foram definidas hipóteses na verificação de melhoria do grau de coordenação da cadeia couro nacional entre períodos e melhoria da coordenação de couro da região Centro-Oeste. Todas as hipóteses foram confirmadas, desse modo, é possível afirmar que a utilização do coeficiente de variação da produção anual de couro é um proxy adequado para se avaliar o grau de coordenação de cadeias.
\end{abstract}

Cristiano Moreira Raimundo

Universidade de Brasília

cristiano.raimundo@gmail.com

Karim Marini Thomé

Universidade de Brasília

thome@unb.br

Recebido em: 18/08/2017. Aprovado em: 24/07/2017

Avaliado pelo sistema double blind review

Avaliador científico: Daniel Carvalho de Rezende

DOI: 10.21714/2238-68902017v19n2p126

\begin{abstract}
The objective of this study is to characterize the coordination performance in the leather industry by measuring the variability of the amount of leather received and purchased for tanning, in the years from 1999 to 2012. For this, the Transaction Cost Theory was used as analytical framework to discuss the coordination and the governance structure of the Brazilian leather industry. The data were extracted from the quarterly census conducted by the Brazilian Institute of Geography and Statistics (IBGE). Hypotheses were set to verify the improvement in the degree of coordination of the Brazilian leather chain during this period, and the improvement of leather coordination in the Center-West region. All hypotheses were confirmed. Thus, it is possible affirm that the use of the coefficient of variation of the annual leather production is a suitable proxy for assessing the coordination degree of the chain.
\end{abstract}

Palavras-chave: Couro, Coordenação, Brasil.

Keywords: Leather, Coordination, Brazil.

\section{INTRODUÇÃO}

A indústria do couro é uma das mais antigas e tradicionais no Brasil, e apresenta um market share de $13 \%$ do mercado mundial. A grande oferta de matériaprima para a produção e um parque industrial detentor de tecnologia e mão de obra especializada são fatores importantes para a competitividade brasileira (CENTRO DAS INDÚSTRIAS DE CURTUME DO BRASIL, 2013; VIANA; FAGUNDES, 2013).

Em 11 anos, o setor curtidor registrou aumento de $465 \%$ no porcentual da receita obtida, passando de US\$ 318 milhões, em 2000, para US\$ 1,48 bilhão, em 2011. As exportações brasileiras de couros e peles, com base no balanço da Secretaria de Comércio Exterior (Secex) do MDIC, movimentaram, em 2011, US\$ 2,05 bilhões, totalizando 352,2 mil toneladas, o que corresponde ao aumento de $17 \%$ em relação a 2010, quando o setor apurou US\$ 1,74 bilhão (CENTRO DAS INDÚSTRIAS DE CURTUME DO BRASIL, 2013; VIANA; FAGUNDES, 2013).

Os esforços da indústria curtidora, somado ao trabalho associativo das empresas de curtumes, têm trazido significantes resultados para o Brasil. No balanço das exportações do mês de abril de 2013, registrou-se o recorde histórico de vendas de couros ao mercado externo em um único mês: US\$218,438 milhões, ou seja, uma alta de 24,6\% em relação ao mesmo mês de 2012 (CENTRO DAS INDÚSTRIAS DE CURTUME DO BRASIL, 2013).

No entanto, apesar dos expressivos números e evoluções relatados, a demanda crescente pelo uso do 
couro bovino não tem sido acompanhada, na mesma proporção, pela presença de uma matéria-prima de qualidade. Jacinto et al. (2012) relatam que o grande entrave para o desenvolvimento da cadeia produtiva de couro brasileira é a baixa qualidade da pele bovina, devido, principalmente, à ocorrência de ectoparasitoses (berne, carrapato e outros), arranhões e marcas a fogo, acarretando, assim, uma condição de subpreço.

Diante desse cenário, faz-se necessário produzir uma matéria-prima de melhor qualidade e desonerar a produção com a retomada do programa de substituição das exportações de couro sob a forma de wet-blue por couros semiacabados e acabados, que apresentam uma maior valorização por unidade comercializada (CAMPOS; LIRIO, 2007).

Além da baixa qualidade, salienta-se que o mercado de couro cru bovino está subordinado ao mercado de carne bovina que, por sua vez, depende de uma série de fatores como o nível de poder de compra, a demanda do mercado internacional, o preço das carnes substitutas, e da estocagem, além de epidemias e fenômenos climáticos como a estiagem, que podem diminuir a oferta de couro com reflexos sobre o preço (CORRÊA, 2001).

Esse cenário permeado de incertezas caracterizase pelo fato do mercado de couro ser predominantemente regido pelo mercado spot, no qual o principal mecanismo de coordenação é o preço, em que não há garantias. As exigências de qualidade são menores, a visão de lucro é de curto prazo, a identidade dos atores tem pouca importância, acarreta o grau baixo de coordenação, pode levar à ocorrência de comportamentos oportunistas e ações ocultas (SLANGEN; LOUCK; SLANGEN, 2008; WILLIAMSON, 1975).

Em resposta a esse cenário de instabilidade e imprevisibilidade, Souza (2002) afirma que a redução da incerteza pode ser obtida por meio de uma relação mais coordenada na comercialização, remuneração pela qualidade e continuidade nas transações.

Já Mielle e Coldebella (2010) acrescentam que os esforços de melhoria do grau de coordenação perseguem vários objetivos, e que o aumento da estabilidade do fluxo de matérias-primas é um dos mais importantes, devido à redução do tempo ocioso.

Portanto, o objetivo deste estudo é de caracterizar o desempenho da coordenação do mercado de couro cru bovino brasileiro entre os anos de 1999 a 2012, pela medição da variabilidade da quantidade de couro inteiro de bovino cru adquirida e recebida de terceiros para curtimento, utilizando o coeficiente de variação como um possível proxy, testando-o e analisando-o sob diferentes hipóteses (modelos empíricos regionais e temporais).

\section{REFERENCIAL TEÓRICO}

\subsection{Aspectos Produtivos de Couro nas Regiões do Brasil}

Ruppenthal (2001) destaca que o setor coureiro representa uma relevante parcela da economia com capacidade de atuar em novos mercados e com grande capacidade para aumentar a produtividade da cadeia em parceria com a pecuária e a indústria manufatureira, com melhoria na qualidade do produto.

Segundo Azevedo e Vinholis (2000), a cadeia produtiva de couro e derivados inicia-se, de fato, na atividade de bovinocultura, em que os diferentes sistemas de criação podem resultar em peles de qualidades distintas, impondo restrições ao processamento do couro e derivados.

Os curtumes se encarregam do processamento do couro animal com o objetivo de fornecer, por exemplo, o couro acabado para a fabricação de diversos produtos finais, como calçados, móveis e estofamentos de automóveis.

De acordo com Costa (2002), a estrutura industrial do setor de curtumes brasileiro é formada por empresas de diferentes tamanhos, predominando estabelecimentos de micro e pequeno porte em termos numéricos. Em 2000, com dados da Relação Anual de Informações Sociais (RAIS) do Ministério do Trabalho e Emprego, 90\% do total de estabelecimentos eram micros e pequenas empresas e eram responsáveis por 39,3\% do emprego do setor. A estrutura produtiva localiza-se, principalmente, em São Paulo e no Rio Grande do Sul, que juntos possuem metade dos estabelecimentos curtumeiros e cerca de $60 \%$ do emprego do setor.

Os curtumes no Brasil constituem um mercado pouco concentrado, dada a presença de inúmeras empresas, com participações modestas no mercado. Como consequência, a indústria de curtumes é bastante fragmentada e heterogênea. No entanto, esse padrão de estrutura de mercado não é observado quando se tem como referência a participação nas exportações, uma vez que os curtumes de pequeno porte têm uma pequena participação no total das exportações (AZEVEDO; VINHOLIS, 2000).

O processamento do couro passa basicamente por seu curtimento e acabamento nos curtumes, os quais podem ser classificados de acordo com a etapa de processamento do couro que realizam: (a) o curtume wet blue desenvolve o primeiro processamento do couro após o abate, quando é dado o primeiro banho de cromo, gerando um tom 
azulado e molhado do couro (wet blue); (b) o curtume de semiacabado utiliza o couro wet blue como matériaprima e o transforma em couro crust (semiacabado); (c) o curtume de acabamento transforma o couro crust em couro acabado; e (d) o curtume integrado realiza todas as operações de processamento, desde o couro cru até o acabado (CUNHA, 2011).

Conforme dados do Instituto Brasileiro de Geografia e Estatística (IBGE), em 1999, a quantidade de couro adquirida e recebida para curtimento foi de 5,8 milhões de unidade, a região Sul apresentava-se como a região de maior destaque com 2,5 milhões de unidades $(43,75 \%$ da produção nacional). Em 2012, esse valor aumentou para 9,1 milhões de unidades, ou seja, um aumento de 56,90\% referente a 1999. E, também houve uma mudança de liderança mercadológica regional, em que a região CentroOeste apresentou-se como a região de maior produção nacional com 4,8 milhões de unidades ( $56,85 \%$ da produção nacional), como visualizado na Figura 1.

\subsection{Teoria da Economia dos Custos de Transação}

A Economia de Custos de Transação (ECT) teve sua origem no campo do direito, economia e administração, em meados dos anos 30. Segundo Arrow (1969) e Williamson (1993), os custos de transação são os custos da administração do sistema econômico. Para o autor, os custos devem distinguir-se dos custos de produção, que é a categoria de custos abordados nas análises neoclássicas.

Essa teoria abrange um enfoque microanalítico para o estudo da organização econômica, centrando sua atenção nas transações que ocorrem dentro da organização. Nesse sentido, a ECT passa a ser amplamente aplicada para análises de relações envolvendo investimentos em ativos específicos, tendo em vista que o objetivo é o de estudar os custos de transação como indutor de modos alternativos de organização da produção (governança), dentro de um arcabouço analítico institucional. Portanto, a transação (operação na qual são negociados direitos de propriedade) passa, então, a ter papel fundamental como unidade de análise (WILLIAMSON, 2003).

Para Williamson (1993), a ECT difere da teoria econômica neoclássica por alguns pressupostos importantes ora ignorados, um deles está ligado ao comportamento dos indivíduos, que podem apresentar racionalidade limitada e oportunismo.

Segundo Williamson (1993), a racionalidade limitada ao comportamento que pretende ser racional, mas consegue sê-lo apenas de forma limitada, resulta da condição de competência cognitiva limitada de receber, estocar, recuperar e processar a informação. Todos os contratos complexos são inevitavelmente incompletos devido à racionalidade limitada.

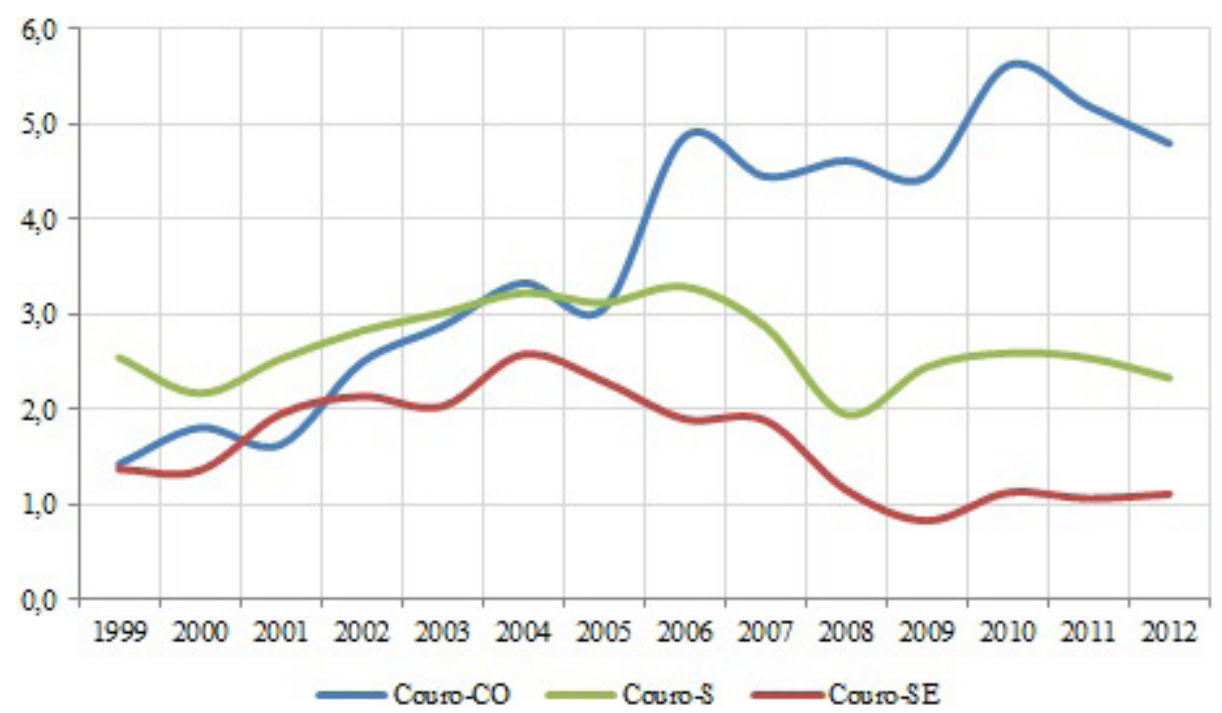

FIGURA 1 - Número de milhões de unidades de couro recebidas para curtimento nas regiões Centro-Oeste (CO), Sudeste (SE) e Sul (S) de 1999 a 2012

Fonte: Instituto Brasileiro de Geografia e Estatística (2013a)

Organizações Rurais \& Agroindustriais, Lavras, v. 19, n. 2, p. 126-136, 2017 
O oportunismo é o outro pressuposto comportamental, é um conceito que resulta da ação dos indivíduos na busca do seu autointeresse. Entretanto o autointeresse pode ser buscado de maneira não oportunista. Afinal, segundo Williamson (1993, 2003), oportunismo parte de um princípio de jogo não cooperativo, no qual a informação que um agente possa ter sobre a realidade não acessível a outro agente pode permitir que o primeiro desfrute de algum benefício do tipo monopolístico.

Williamson (2003) apresenta três características básicas das transações, categorizadas por Williamson (1975) como: frequência, incerteza e especificidade de ativos. A característica frequência refere-se ao número de vezes que uma transação se repete, em transações repetitivas pode haver a construção de uma reputação, reduzindo questões ligadas ao oportunismo e à incerteza, reduzindo os custos de transação.

Já a incerteza implica na impossibilidade de antecipação dos acontecimentos futuros, pode ocasionar rompimento contratual não oportunístico e está associada ao surgimento de custos de transação. A especificidade dos ativos refere-se quando o ativo é desenvolvido para uso determinado, caso este não ocorra, não há uso alternativo sem perda de seu valor. Quanto maior a especificidade dos ativos maior serão os custos de transação.

\subsection{Coordenação}

A coordenação não é uma característica intrínseca dos sistemas produtivos, mas sim o resultado de uma construção dos agentes econômicos. Conforme Thomé, Reis e Paiva (2013), a existência de coordenação não quer dizer que não existam conflitos distributivos potenciais ou efetivos, nem a ausência de contratos formais. A existência de sistemas ineficientes de coordenação beneficia alguns agentes e pode representar forças de resistência a mudanças.

Segundo Slangen, Louck e Slangen (2008), os mecanismos de coordenação podem ser classificados em quatro grupos: (1) "mão invisível"; (2) "mão visível”; (3) "manual"; (4) "aperto de mão".

No grupo "mão invisível", o grupo de mecanismo é o preço e no grupo "mão visível", a autoridade e supervisão direta. Já no grupo "manual”, o grupo de mecanismo são as leis, diretrizes e salvaguardas. Enquanto que no grupo "aperto de mão", o grupo de mecanismo é o ajuste mútuo e valores comuns e normas (SLANGEN; LOUCK; SLANGEN, 2008).

Nesse sentido, para Thomé, Reis e Paiva (2013), havendo um sistema de coordenação ineficiente, existe o aumento das possibilidades de atitudes oportunistas dos agentes, o que justifica, muitas vezes, o interesse destes para que não exista uma coordenação eficiente na cadeia produtiva ou em seus elos específicos.

Segundo Wacker, Yang e Sheu (2016), a coordenação eficiente da cadeia é ponto fundamental para o sucesso das estratégias adotadas, tendo em vista que ela deve ser construída a partir do desenvolvimento de instituições capazes de reduzir os custos associados às transações em adequação às condições presentes no ambiente geral.

A capacidade de aproveitar as oportunidades depende de um sistema de coordenação capaz de transmitir informações, estímulos e controles ao longo da cadeia produtiva para viabilizar estratégias adotadas. É importante que os participantes da cadeia agroindustrial também articulem estratégias para atingir seus objetivos (LEONELLI; OLIVEIRA, 2016; THOMÉ; VIEIRA; SANTOS, 2012).

\subsection{Estruturas de Governança}

Segundo Gibbons (2010), Williamson (1975) propõe que a firma, vista como uma estrutura de governança das transações, pode optar por tratar determinado contrato a partir de uma relação somente de mercado ou por uma forma mista contratual ou, ainda, pela necessidade de integração vertical, motivada pelos princípios de minimização dos custos de produção, somados aos custos de transação.

Slangen, Louck e Slangen (2008) afirmam que as estruturas de governança emergem em resposta a várias considerações transacionais, acarretando assim na escolha do melhor arranjo institucional, conforme os mecanismos de coordenação vigentes.

Vilpoux (2011), num estudo sobre desempenho de arranjos institucionais de produtores de mandioca e fecularias, propõe a classificação dos seis grupos de estrutura de governança, de acordo com o nível de garantias oferecidas em relação ao fornecimento de matéria-prima: (1) mercado spot (não oferece nenhuma garantia aos intervenientes); (2) mercado com garantias informais (transações no mercado entre atores que se conhecem e que mantêm contatos regulares); (3) acordos contratuais com garantias fracas (contratos elaborados podem ser formais ou não, mas sempre se caracterizam pelos baixos níveis de garantias); (4) acordos contratuais com garantias intermediárias (como no grupo “3”, esses contratos podem ser formais ou não, mas a probabilidade de respeitar o acordo é maior); (5) acordos contratuais com garantias fortes (também chamados de parceria, utilizando contratos que garantam "escoamento" da produção e medidas que eliminem ações oportunísticas pelos atores); (6) integração vertical (todas as etapas de produção são realizadas pelo empresário). 
Os sistemas de governança diferenciam-se, de acordo com as garantias oferecidas que podem ser formais ou informais. As governanças " 3 " e "4" apoiam-se em garantias que misturam essas características. A baixa eficácia dessas garantias não permite grande segurança nos compromissos assumidos, obtida em contratos com garantias mais fortes, sejam formais ou não (estrutura " 5 "), e na integração vertical. Em função do grau de confiança entre os atores, o modo de coordenação no mercado e com garantias informais ("2") aproxima-se do mercado ("1") ou dos acordos contratuais com garantias fracas (“3”). As governanças via hierarquia (“6”) são aquelas que oferecem os maiores níveis de garantia.

As formas eficientes de governança a serem adotadas devem considerar os riscos como a racionalidade limitada, os contratos incompletos, a presença de atitudes oportunistas e a existência de especificidade de ativos. Além disso, deve-se considerar o grau dessa especificidade, pois no caso desta ser alta, há estímulos para a adoção da integração vertical na produção da empresa (LEONELLI; OLIVEIRA, 2016; PAULILLO; NEVES, 2015).

A coordenação eficiente construída pelos agentes econômicos na cadeia é fundamental para o sucesso das estratégias adotadas por estes, por isso os agentes devem tomar consciência disso (THOMÉ; VIEIRA; SANTOS, 2012).

\subsection{Avaliação do Grau de Desempenho da Coordenação e Hipóteses Levantadas}

Gibbons (2010) destaca as críticas sofridas pela ECT a respeito da falta de modelos formais. Esse autor ainda acrescenta que o avanço conceitual da ECT depende da formulação de modelos formais que traduzam os conceitos da ECT em variáveis mensuráveis e passíveis de testes. Assumindo assim, que o avanço técnico não tem acompanhado o avanço teórico nessa teoria de significativa relevância e aderência ao agronegócio brasileiro.

Miele e Zylbersztajn (2005), antes mesmo dos comentários de Gibbons (2010), propuseram avaliar o grau de coordenação de viticultores e vinícolas na Serra Gaúcha, pela mediação da variação de processamento de empresas, em dois períodos de anos. Nessa mesma linha, Miele e Coldebella (2010) propuseram a utilização do coeficiente de variação (CV) do abate anual de frangos, suínos e bovinos como um proxy para se avaliar o grau de coordenação desses complexos.

Por meio desta metodologia, da utilização do CV, pode-se mensurar o grau de desempenho da coordenação de um determinado complexo de forma objetiva e numérica. Aplicando este proxy no presente estudo, e tendo como base o alinhamento produtivo pregado na relação comercial que é visto na coordenação de Menard (2002), pode-se concluir que a obtenção de um alto CV sugere um baixo desempenho de coordenação, em que os produtores de couro acabado (abatedouros, curtumes e salgadores) estariam em busca de uma estabilização produtiva. Em contrapartida, um CV baixo sugere um melhor desempenho de coordenação.

A priori, a grande mudança de cenário competitivo entre regiões produtoras de couro no Brasil caracterizada pela estagnação do número de unidades de couro recebidas para curtimento na região Sul e o seu respectivo expressivo aumento na região Centro-Oeste brasileira, principalmente a partir de 2005, como ilustra a Figura 1, demanda uma avaliação particular do grau de coordenação da produção de couro, tomando a região Centro-Oeste como centro das análises e em dois períodos distintos.

Conforme destacado por Williamson (1985), a coordenação não é uma característica intrínseca dos sistemas produtivos, mas, sim, resultado de uma construção dos agentes econômicos, com a finalidade de reduzir os custos de transação, por meio da adoção de estruturas de governança apropriadas. Gulbrandsen, Lambe e Sandvik (2017) complementam a proposição de Williamson (1985) ao afirmar que quanto mais apropriada for a coordenação entre os componentes do sistema, menores serão os custos de cada um deles, mais rápida será a adaptação às modificações de ambiente e menos custosos serão os conflitos inerentes às relações cliente/ fornecedor (LEONELLI; OLIVEIRA, 2016).

Logo, com base nas proposições de Gulbrandsen, Lambe e Sandvik (2017) e Williamson (1985), faz-se importante entender se a maior competitividade da região Centro-Oeste, principalmente a partir de 2005, quando esta região se tornou a maior curtidora de unidades de couros do Brasil, está associada a um maior grau de coordenação dessa cadeia nessa região. Essa avaliação pode indicar se a região Centro-Oeste está apresentando melhores formas de organização de suas transações econômicas em relação às demais regiões nacionais, por meio de uma organização mais eficiente de toda a sua cadeia de produção, indicada por um menor CV de produção desse produto, ao longo dos anos.

Dessa forma, para se avaliar o grau de coordenação pelo $\mathrm{CV}$, definiram-se hipóteses (Quadro 1) sugerindo modelos empíricos a serem testados, no sentido de melhoria do grau de coordenação da produção de couro ao longo dos anos, conforme mudanças mercadológicas de abate de bovinos e produção de couro nas regiões CentroOeste (CO), Sudeste (SE) e Sul (S) presente nos dados do IBGE (INSTITUTO BRASILEIRO DE GEOGRAFIA E ESTATÍSTICA, 2013b) e citadas na seção 2.1 deste estudo. As hipóteses formuladas com base na evolução da coordenação nacional e em regiões específicas está no Quadro 1 apresentado na metodologia. 
QUADRO 1 - Hipóteses a serem testes e confirmadas e suas respectivas descrições qualitativas

\begin{tabular}{|c|c|}
\hline Hipóteses: & Descrição qualitativa da hipótese: \\
\hline $\mathrm{H}_{1}: \mathrm{CV}_{\mathrm{CO} ; \mathrm{SE} ; \mathrm{S} \text { (Período "1") }}>\mathrm{CV}_{\mathrm{CO} ; \mathrm{SE} ; \mathrm{S} \text { (Período "2") }}$ & $\begin{array}{l}\text { Houve evolução da coordenação da cadeia nacional de couro } \\
\text { cru bovino entre os períodos em análise; }\end{array}$ \\
\hline $\mathrm{H}_{2}: \mathrm{CV}_{\mathrm{CO} \text { (Período "1") }}>\mathrm{CV}_{\mathrm{SE} \text { (Periodo "1") }}>\mathrm{CV}_{\mathrm{S} \text { (Período "1") }}$ & $\begin{array}{l}\text { O desempenho de coordenação da cadeia couro é menor da na } \\
\text { região CO que das regiões SE e S no período "1". }\end{array}$ \\
\hline $\mathrm{H}_{3}: \mathrm{CV}_{\mathrm{CO} \text { (Período "2") }}<\mathrm{CV}_{\mathrm{S} \text { (Período "2") }}<\mathrm{CV}_{\mathrm{SE} \text { (Período 2) }}$ & $\begin{array}{l}\text { O desempenho de coordenação da cadeia couro é maior da na } \\
\text { região CO que nas regiões S e SE no período de "2". }\end{array}$ \\
\hline $\mathrm{H}_{4}: \Delta \mathrm{CV}_{\mathrm{CO}}<\Delta \mathrm{CV}_{\mathrm{S}}<\Delta \mathrm{CV}_{\mathrm{SE}}$ & $\begin{array}{c}\text { A evolução da coordenação da região CO é maior que nas } \\
\text { regiões } \mathrm{S} \text { e SE. }\end{array}$ \\
\hline
\end{tabular}

Fonte: Elaborado pelos autores

\section{METODOLOGIA}

A metodologia utilizada neste estudo utiliza técnicas quantitativas e qualitativas para analisar os dados provenientes da Pesquisa Trimestral de Couro conduzida pelo IBGE em 2013. Essa pesquisa possui os dados coletados do número de unidades de couro inteiro bovino cru adquirido e recebido de terceiros para curtimento de 1999 a 2012, em todos os 27 estados brasileiros.

Os dados foram filtrados e agrupados em 3 regiões. A região Sudeste (SE) contém os estados do Espírito Santo (ES), São Paulo (SP) e Minas Gerais (MG); a região Sul (S) contém os estados do Paraná (PR), Santa Catarina (SC) e Rio Grande do Sul (RS); a região Centro-Oeste (CO) contém os estados do Mato Grosso (MT), Mato Grosso do Sul (MS) e Goiás (GO). Essas três regiões representam respectivamente $12,50 \% ; 26,33 \% ; 54,29 \%$ do número de unidades de couro inteiro bovino cru adquirido e recebido de terceiros de para curtimento, totalizando assim, $93,13 \%$ da produção nacional de 2012 (INSTITUTO BRASILEIRO DE GEOGRAFIAE ESTATÍSTICA, 2013a).

Primeiramente, para se definir os períodos (conjunto de anos) a serem comparados os graus do desempenho de coordenação, foi realizada uma série de regressões múltiplas da produção de couro (variável dependente $\mathrm{Y}$ ) em função dos anos (variável independente contínua $\mathrm{X}_{1}$ ) e período (variável dicotômica " 0 " ou " 1 " independente $\mathrm{X}_{2}$ ) em nível nacional. Os dois períodos selecionados foram aqueles que obtiverem a regressão significativa a $5 \%$, conforme teste $\mathrm{F}$ e os coeficientes estimados das variáveis $\mathrm{X}_{1}$ e $\mathrm{X}_{2}$ com significância de $5 \%$, conforme teste " $\mathrm{t}$ " de Student. Caso verificado mais de uma simulação com coeficientes estatisticamente significativos definiu-se como critério de escolha os períodos com número de anos mais próximos entre si, buscando um melhor equilíbrio entre os períodos, sugerindo uma melhor extração da variabilidade.
Adicionalmente, com o objetivo de se identificar uma estabilidade nacional da produção de couro, foram calculadas taxas de crescimento nacional nos períodos pré-definidos, conforme metodologia proposta por Negri Neto, Coelho e Moreira (1993) em que certa grandeza tenha valor $\mathrm{V}_{0}$ num certo ano, que a taxa anual de crescimento seja r. Generalizando este conceito para vários anos:

$V_{n}=V_{0}(1+r)^{n}$

Aplicando o logaritmo natural (Ln) na equação acima, tem-se que:

$\operatorname{Ln} V_{n}=\operatorname{Ln} V_{0}+n \operatorname{Ln}(1+r)$

A equação acima é similar a uma equação de regressão linear de modelo:

$Y=a X+b$

Ao isolar $r$, tem-se que:

$r=\left[\operatorname{antilog}\left(L n V_{n}-L_{n} V 0\right) / n\right]-1$

As taxas de crescimento (r) foram analisadas se são estatisticamente significantes a um nível de probabilidade de 5\%, conforme teste $\mathrm{F}$ do quadro de análise de variância das regressões.

Para obter-se a medida de variabilidade, foi calculado o coeficiente de variação $(\mathrm{CV})$ dos períodos definidos. O CV é definido como a razão entre o desvio padrão populacional $(\sigma)$ e a média populacional $(\mu)$ do número de unidades de couro, e a sua equação é $\mathrm{CV}=\frac{\sigma}{\mu} 100$. Neste estudo, o $\mathrm{CV}$ do número de unidade de couro cru bovino recebido e adquirido pode representar uma proposta de um indicador que meça o desempenho da coordenação da cadeia de produtiva de couro brasileiro. 


\section{RESULTADOS}

Conforme a Tabela 1, as simulações "07" e "08" apresentaram-se estatisticamente significativas nos pressupostos definidos neste estudo. Porém, foram selecionados os períodos da simulação "07", por esta simulação possuir o número de anos por período mais equilibrado entre as simulações realizadas. Portanto, as análises deste estudo serão em relação ao período 1 (1999;
2000; 2001; 2002; 2003; 2004; 2005; 2006) e período 2 $(2007 ; 2008 ; 2009 ; 2010 ; 2011 ; 2012)$.

Sendo:

$\mathrm{A}=$ Significativo a um nível de probabilidade de 5\%;

$\mathrm{B}=$ Não significativo a um nível de probabilidade de $5 \%$.

Ao se analisar separadamente os períodos, Figura 2, verificou-se que no período 1 (de 1999 a 2006), a taxa média de crescimento anual foi de $9,44 \%$, a um nível de probabilidade de 5\%. Enquanto que no período 2 (de 2007

TABELA 1 - Probabilidade do teste F dos modelos de regressão (Prob. Reg.) nos períodos simulados com diferentes anos, probabilidades das estatísticas t de Student dos coeficientes angulares de $\mathrm{X}_{1}$ e $\mathrm{X}_{2}$ (Prob. Coef.)

\begin{tabular}{|c|c|c|c|c|c|}
\hline Simulação & Anos Período 1 & Anos Período 2 & Prob. Reg. & Prob. Coef. $X_{1}$ & Prob. Coef. $\mathrm{X}_{2}$ \\
\hline 01 & $1999-2000$ & $2001-2012$ & $0,004^{\text {sig.A }}$ & $0,206^{\text {nsig.B }}$ & $0,028^{\text {sig. }}$ \\
\hline 02 & 1999-2001 & 2002-2012 & $0,000^{\text {sig. }}$ & $0,779^{\text {nsig. }}$ & $0,002^{\text {sig. }}$ \\
\hline 03 & 1999-2002 & $2003-2012$ & $0,001^{\text {sig. }}$ & $0,988^{\text {nsig. }}$ & $0,009^{\text {sig. }}$ \\
\hline 04 & $1999-2003$ & 2004-2012 & $0,011^{\text {sig. }}$ & $0,763^{\text {nsig. }}$ & $0,096^{\text {nsig. }}$ \\
\hline 05 & 1999-2004 & $2005-2012$ & $0,044^{\text {sig. }}$ & $0,221^{\text {nsig. }}$ & $0,837^{\text {nsig. }}$ \\
\hline 06 & 1999-2005 & 2006-2012 & $0,044^{\text {sig. }}$ & $0,130^{\text {nsig. }}$ & $0,822^{\text {nsig. }}$ \\
\hline 07 & $1999-2006$ & $2007-2012$ & $0,004^{\text {sig. }}$ & $0,002^{\text {sig. }}$ & $0,033^{\text {sig. }}$ \\
\hline 08 & $1999-2007$ & $2008-2012$ & $0,000^{\text {sig. }}$ & $0,000^{\text {sig. }}$ & $0,000^{\text {sig. }}$ \\
\hline 09 & 1999-2008 & 2009-2012 & $0,007^{\text {sig. }}$ & $0,003^{\text {sig. }}$ & $0,063^{\text {nsig. }}$ \\
\hline 10 & 1999-2009 & $2010-2012$ & $0,002^{\text {sig. }}$ & $0,013^{\text {sig. }}$ & $0,275^{\text {nsig. }}$ \\
\hline 11 & $1999-2010$ & $2001-2012$ & $0,016^{\text {sig. }}$ & $0,006^{\text {sig. }}$ & $0,159^{\text {nsig. }}$ \\
\hline
\end{tabular}

Fonte: Resultado da pesquisa

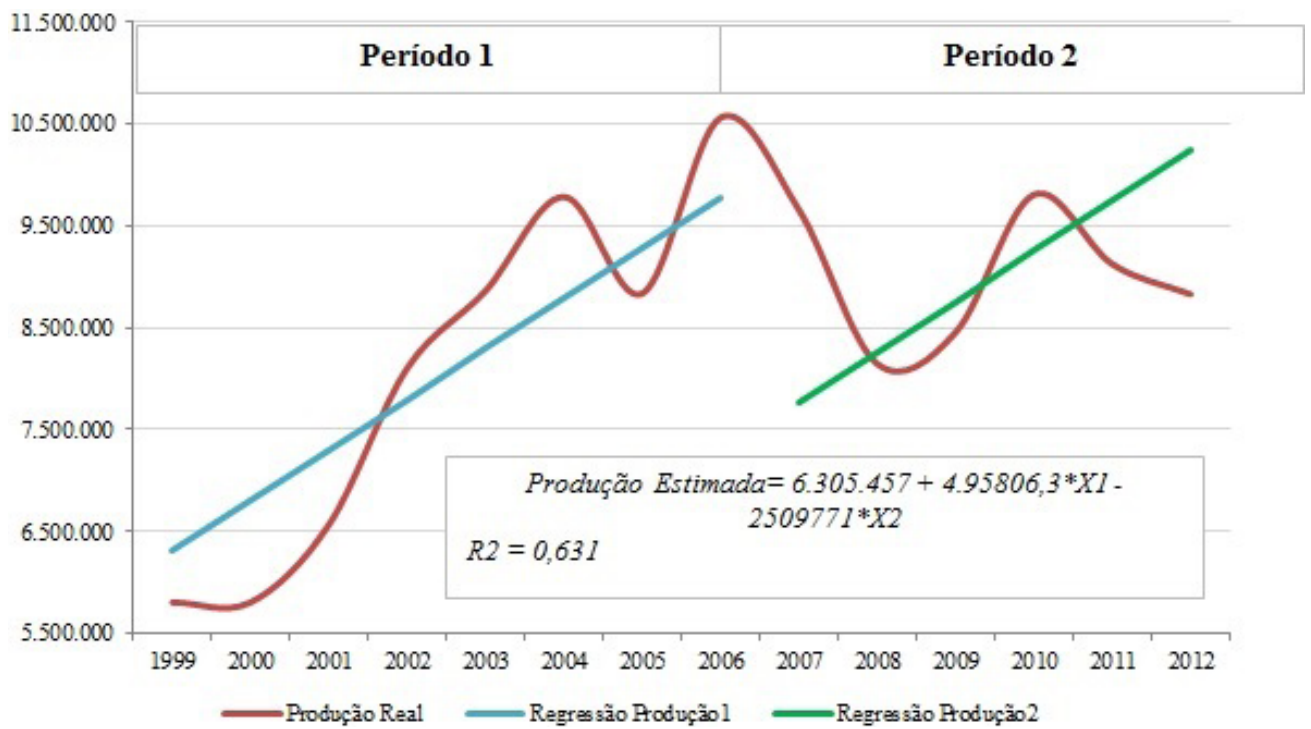

FIGURA 2 - Gráfico da equação de regressão linear múltipla do modelo selecionado período 1 de 1999 a 2005 e período 2 de 2006 a 2012

Fonte: Resultado da pesquisa

Organizações Rurais \& Agroindustriais, Lavras, v. 19, n. 2, p. 126-136, 2017 
a 2012), a taxa de crescimento estatisticamente não se diferiu de $0 \%$ a um nível de probabilidade de $5 \%$, conforme visto na Tabela 2, sugerindo uma estabilidade produtiva e, consequentemente, menor variabilidade do setor nacional de couros no segundo período.

Os resultados apresentados neste estudo não rejeitam as quatro hipóteses formuladas para este estudo nos dois períodos definidos previamente.

TABELA 2 - Probabilidade da regressão (Prob. Reg.), taxa de crescimento médio percentual do período (r) e probabilidade das estatísticas t de Student do coeficiente angular de $r$

\begin{tabular}{cccc}
\hline Período & Prob. Reg. & r (\%) & Prob. Coef. r \\
\hline $1999-2006$ & $0,000^{\mathrm{A}}$ & 9,44 & $0,000^{\mathrm{A}}$ \\
$2007-2012$ & $0,955^{\mathrm{B}}$ & 0,12 & $0,955^{\mathrm{B}}$ \\
\hline
\end{tabular}

Fonte: Elaborado pelos autores

Sendo:

$\mathrm{A}=$ Significativo a um nível de probabilidade de 5\%;

$\mathrm{B}=$ Não significativo a um nível de probabilidade de $5 \%$.

Fonte: Resultado da pesquisa
Considerando a hipótese 1 , o CV da soma das três regiões, que representam $93,13 \%$ do curtimento nacional de couro cru bovino, apresentou uma queda comparando o primeiro período dos dados (1999 a 2006) em relação ao segundo (2007 a 2012). Houve uma redução do CV de 21,03 para 6,66 , representando uma variação negativa percentual de 68,34. Portanto, os dados sugerem uma melhoria do desempenho de coordenação do mercado de couro cru bovino ao longo os períodos em análise, conforme expresso na Tabela 3.

Conforme proposto na hipótese 2 , no primeiro período em análise, a região $\mathrm{CO}$ apresentou um $\mathrm{CV}$ de 39,33, o maior entre as regiões. A região $S$ apresentou o menor CV de 13,04, o menor entre as regiões. A região SE apresentou um CV de 18,67, foi o intermediário (Tabela 3).

Considerando a hipótese 3 , que sugere uma grande evolução no desempenho da coordenação dos estados do $\mathrm{CO}$ no segundo período de análise, constatou-se que a região $\mathrm{CO}$ apresentou um $\mathrm{CV}$ de 8,78, o menor dentre as regiões. A região $\mathrm{SE}$ apresentou um $\mathrm{CV}$ de 18,67, o maior entre as regiões. A região $\mathrm{S}$ apresentou um $\mathrm{CV}$ de 13,04, foi o intermediário (Tabela 3). Essa melhoria do desempenho da coordenação do mercado de couro na região $\mathrm{CO}$, quando se compara as hipóteses 2 com a 3 ,

TABELA 3 - Coeficientes de variação (CV) e participações percentuais (Partic.) da produção de couro bovino cru dos principais estados e regiões brasileiras, em dois períodos de anos (1999 a 2006; 2007 a 2012)

\begin{tabular}{|c|c|c|c|c|c|}
\hline Regiões & $\mathrm{CV}_{1999-2006}$ & Partic. ${ }_{1999-2006}$ & $\mathrm{CV}_{2007-2012}$ & Partic. ${ }_{2007-2012}$ & $\Delta \mathrm{CV}$ \\
\hline MS & 22,14 & 16,70 & 6,91 & 13,16 & $-68,78$ \\
\hline GO & 77,61 & 9,80 & 18,65 & 13,40 & $-75,97$ \\
\hline MT & 48,15 & 6,81 & 27,78 & 18,65 & $-42,78$ \\
\hline $\mathrm{CO}$ & 39,33 & 33,31 & 8,78 & 45,21 & $-77,68$ \\
\hline RS & 5,65 & 25,43 & 17,47 & 16,73 & 209,12 \\
\hline PR & 39,83 & 9,67 & 45,07 & 6,10 & 13,15 \\
\hline $\mathrm{SC}$ & 96,96 & 0,14 & 223,61 & 0,01 & 130,61 \\
\hline $\mathrm{S}$ & 13,04 & 35,24 & 11,49 & 22,84 & $-11,94$ \\
\hline SP & 19,94 & 21,32 & 32,04 & 8,93 & 60,71 \\
\hline $\mathrm{MG}$ & 112,97 & 0,52 & 109,42 & 1,50 & $-3,15$ \\
\hline ES & 264,57 & 0,51 & -1 & 0,00 & - \\
\hline $\mathrm{SE}$ & 18,67 & 22,34 & 18,90 & 10,44 & 1,24 \\
\hline Total & 21,03 & 100,00 & 6,66 & 100,00 & $-68,34$ \\
\hline
\end{tabular}

${ }^{1}$ Não houve produção informada do estado do ES no período de 2006 a 2012, consequentemente, não gerando informações para o cálculo do CV do estado e não havendo na participação da produção total

Fonte: Resultado da pesquisa 
pode ser justificada pela intensificação da concentração de couro pelos grandes frigoríficos devido ao crescimento do processo de integração vertical da produção de bovinos, enquanto que os altos CV's das regiões S e SE podem ser justificados pela ainda predominância de empresas de menor porte, caracterizando-se por uma atomização dos produtores, ou seja, na cadeia de couro nacional, ainda coexiste um elevado número de pequenos e médios curtumes com um grupo reduzido de grandes curtumes e grandes frigoríficos verticalmente integrados (CUNHA, 2011). Colaborando com essa justificativa, cita-se que, em 2009, as regiões S e SE apresentaram, respectivamente, um total de 243 e 281 empresas, enquanto que a região CO apresentou apenas 63 empresas, enfatizando o perfil mais concentrado e verticalizado dessa região (MTE, 2009).

Considerando a hipótese 4, a região $\mathrm{CO}$ apresentou uma variação percentual negativa de CV de 77,68, apresentando-se como a maior redução de CV entre as regiões. Todos os estados da região $\mathrm{CO}$ apresentaram reduções negativas percentuais nos CV's. Os estados de MS, GO e MT apresentaram significativas quedas percentuais de $\mathrm{CV}$ entre os períodos em análise, respectivamente, $68,78,75,97$ e 42,78, sugerindo uma contribuição para a melhora do desempenho de coordenação dessa região. A região $\mathrm{SE}$ teve variação percentual positiva de $\mathrm{CV}$ entre os períodos em análise de apenas 1,24 . A região $\mathrm{S}$ teve variação percentual negativa de 11,94 , porém, todos os seus estados tiveram variações percentuais positivas, sugerindo piora no grau de coordenação por estado.

Ao se analisar as regiões, analisando-se qualitativamente, os dados sugerem que pode existir uma associação negativa entre o CV e a participação percentual da produção de couro, ou seja, no primeiro período em análise, a região $\mathrm{S}$ apresentou o menor $\mathrm{CV}(13,04)$ e a maior participação percentual da produção total $(35,04)$, enquanto que a região $\mathrm{CO}$ apresentou o maior $\mathrm{CV}(39,33)$ e uma participação percentual intermediária $(33,31)$. Em contrapartida, no segundo período, a região $\mathrm{CO}$ apresentou o menor CV $(8,78)$ e a maior participação percentual $(45,21)$, enquanto que a região $\mathrm{SE}$ apresentou o maior CV $(18,90)$ e a menor participação percentual $(10,44)$. Esse comportamento pode ser um indicativo primário de que a região que apresentar um baixo $\mathrm{CV}$ tem uma boa possibilidade de assumir maiores participações, indicando uma melhor coordenação (MIELE; COLDEBELLA, 2010) $\mathrm{e}$, consequentemente, potencial liderança no setor nacional.

Buscando compreender a melhoria de coordenação da região $\mathrm{CO}$, realizou-se o teste de correlação linear de Pearson da produção de couro em nível nacional e por regiões, de 1999 a 2012, e do respectivo abate de bovinos dessas regiões (dados extraídos do INSTITUTO BRASILEIRO DE GEOGRAFIA E ESTATÍSTICA, 2013b), buscando verificar se existe uma associação linear entre estas duas cadeias (Tabela 4).

Pelos índices de correlação linear de Pearson, verificou-se um índice de 0,842 , sugerindo que a produção de couro da região $\mathrm{CO}$ está fortemente e diretamente associada ao abate de bovinos dessa mesma região. As regiões $\mathrm{S}$ e SE apresentaram índices menores, respectivamente de 0,218 e 0,107 , sugerindo que o abate de bovinos tem fraca associação com a produção de couro, conforme expresso na Tabela 4.

TABELA 4 - Valores do índice de correlação linear de Pearson entre o abate de bovinos e a produção de couro no Brasil, regiões Centro-Oeste (CO), Sul (S) e Sudeste (SE) de 1999 a 2012

\begin{tabular}{cccc}
\hline Brasil & Região CO & Região S & Região SE \\
\hline 0,886 & 0,842 & 0,218 & 0,107 \\
\hline
\end{tabular}

Fonte: Resultado da pesquisa

Apesar dos indícios de melhoria do desempenho da coordenação do mercado de couro cru bovino para curtimento ao longo dos períodos analisados e principalmente pela melhoria significativa do grau de coordenação da região $\mathrm{CO}$, o seu $\mathrm{CV}$ pode ser considerado alto, quando se comparar ao CV's obtidos por Mielle e Coldebella (2010) do abate de frangos e suínos da região S (respectivamente, 5,65 e 5,91, no período de 2003 a 2009). Essas cadeias caracterizam-se por serem altamente verticalizadas e regidas fortemente por contratos. Ressaltase assim, a necessidade de melhoria da coordenação, pela adoção de contratos de longo prazo.

Por fim, constata-se, em um âmbito geral, que a cadeia de couro, ainda, é regida por mercado spot no qual a identidade dos atores, de acordo com a literatura apresentada, tem pouca importância na transação, e, consequentemente, acarretam incertezas e discrepâncias em seus elos, prejudicando a sua competitividade. Portanto, pode-se afirmar, com base em Williamson (1975), que a cadeia do couro, durante o período estudado, foi regida fortemente pela forma de mercado spot, no qual as ações oportuníssimas e incertezas são mais frequentes. Verificase, com base em Thomé, Vieira e Santos (2012), que para adotar estratégias que minimizem esses comportamentos, novas formas de transações ou novos modelos de negócios 
podem ser utilizados, como no estudo relatado por Paulillo e Neves (2015) na análise da estrutura de governança utilizada para compra de laranja, enfocando arranjos híbridos.

\section{CONCLUSÕES}

Com base nos dados analisados, pode-se concluir que todas as quatro hipóteses estruturadas na teoria dos custos de transação foram confirmadas, ou seja, foi observada melhoria do desempenho de coordenação da cadeia couro nacional entre os períodos analisados. Adicionalmente, observou-se que, no primeiro período temporal analisado, a região Sul apresentou-se como a região de melhor de desempenho de coordenação (menor CV) e, no segundo período analisado, a região CentroOeste apresentou-se como a região de melhor desempenho de coordenação (menor CV). Por último, a região CentroOeste apresentou a maior evolução de coordenação entre as demais regiões, por meio da maior redução percentual de CV apresentada.

Pelos resultados obtidos, sugere-se que, com base no grau de coordenação regional da cadeia de couro, a política industrial presente na produção de couro cru da região Centro-Oeste caracterizada como um conjunto de ações deliberadas de coordenação das atividades empresariais que visa a melhorar o desempenho das firmas em seu conjunto (competitividade privada), apresenta-se mais eficiente quando comparada às demais regiões brasileiras.

Portanto, conclui-se que o CV de produção pode ser utilizado como um proxy para melhor compreender a coordenação entre os elos da cadeia produtiva, aqui analisada na produção brasileira de couro cru brasileira.

\section{REFERÊNCIAS BIBLIOGRÁFICAS}

ARROW, K. J. The organization of economic activity: issues pertinent to the choice of market versus nonmarket allocation. In: THE ANALYSIS AND EVALUATION OF PUBLIC EXPENDITURES: THE PBB-SYSTEM, JOINT ECONOMIC COMMITTEE, 9., 1969, Washington. Congress... Washington: Government Printing Office, 1969. p. 59-73.

AZEVEDO, P. F.; VINHOLIS, M. B. Setor de couro e derivados. In: BATALHA, M. O; SILVA, C. A. (Org.). Estudo sobre a eficiência econômica e competitividade da cadeia agroindustrial da pecuária de corte no Brasil: volume 1. Brasília: Instituto Evaldo Lodi, 2000. p. 272-318.
CAMPOS, S.; LIRIO, V. Avaliação da indústria de processamento de couro bovino em Minas Gerais: enfoque na estrutura, conduta e desempenho. Informe GEPEC, Toledo, v. 11, n. 1, p. 54-69, jan./jun. 2007.

CENTRO DAS INDÚSTRIAS DE CURTUME DO BRASIL - CICB. Relatórios 2013. Brasília: CICB, 2013. Disponível em: <http://www.cicb.org.br>. Acesso em: 1 jul. 2013.

CORRÊA, A. R. O complexo coureiro calçadista brasileiro. BNDES Setorial, Rio de Janeiro, n. 14, p. 65-92, set. 2001.

COSTA, A. da. Estudo da competitividade de cadeias integradas no Brasil: impactos da zona de livre comércio cadeia couro calçados. Campinas: UNICAMP, 2002. 142 p.

CUNHA, A. Relatório de acompanhamento setorial Indústria de Couro. Brasília: ABDI, 2011. 44 p.

FARINA, E. Competitividade e coordenação de sistemas agroindustriais: um ensaio conceitual. Gestão \& Produção, São Carlos, v. 6, n. 3, p. 147-160, dez. 1999.

GIBBONS, R. Transaction-cost economics: past, present, and future? The Scandinavian Journal of Economics, Stockholm, v. 112, n. 2, p. 263-288, June 2010.

GULBRANDSEN, B.; LAMBE, C. J.; SANDVIK, K. Firm boundaries and transaction costs: the complementary role of capabilities. Journal of Business Research, Athens, v. 78, p. 193-203, Sept. 2017.

INSTITUTO BRASILEIRO DE GEOGRAFIA E ESTATÍSTICA - IBGE. Censo da produção de couro bovino de 2012. Brasília: IBGE, 2013a. Disponível em: $<$ http://www.ibge.gov.br>. Acesso em: 1 jul. 2013.

INSTITUTO BRASILEIRO DE GEOGRAFIA E ESTATÍSTICA - IBGE. Censo do abate de bovinos de 2012. Brasília: IBGE, 2013b. Disponível em: <http://www. ibge.gov.br>. Acesso em: 1 jul. 2013.

JACINTO, M. et al. Influência dos defeitos na qualidade intrínseca de couro bovino. São Carlos: Embrapa Pecuária Sudeste, 2012. 32 p.

LEONELLI, F. C. V.; OLIVEIRA, I. R. C. Percepção dos consumidores sobre os açougues gourmet: um estudo multicaso. Organizações Rurais \& Agroindustriais, Lavras, v. 18, n. 1, p. 79-91, 2016. 
MENARD, S. Applied logistic regression analysis. 2. ed. Thousand Oaks: Sage, 2002. 128 p.

MIELE, M.; COLDEBELlA, A. Using Brazilian slaughters as a coordination measure. Revista Política Agrícola, Brasília, DF, v. 19, n. 2, p. 42-47, 2010.

MIELE, M.; ZYLBERSZTAJN, D. Coordenação e desempenho da transação entre viticultores e vinícolas na Serra Gaúcha. Revista de Administração, São Paulo, v. 40, n. 4, p. 330-341, out./dez. 2005.

NEGRI NETO, A.; COELHO, P.; MOREIRA, I. Análise gráfica e taxa de crescimento. Informações Econômicas, São Paulo, v. 23, n. 10, p. 99-108, out. 1993.

PAULILLO, L. F.; NEVES, M. C. Análise das estruturas de governança de compra de laranja dos maiores packing houses do estado de São Paulo: um estudo multicascos sobre arranjos híbridos. Organizações Rurais \& Agroindustriais, Lavras, v. 17, n. 4, p. 461-478, 2015.

RUPPENTHAL, J. E. Perspectivas do setor couro do Estado do Rio Grande do Sul. 2001. 259 f. Tese (Doutorado em Engenharia de Produção) - Universidade Federal de Santa Catarina, Florianópolis, 2001.

SLANGEN, L.; LOUCK, L.; SLANGEN, A. Institutional economics and economic organization theory. Wagningen: Wagningen Academic Publishers, 2008. 431 p.

SOUZA, J. P. de. Gestão da competitividade na cadeia agroindustrial de carne bovina do Estado do Paraná. 2002. 272 f. Tese (Doutorado em Engenharia de Produção) Universidade Federal de Santa Catarina, Florianópolis, 2002.

THOMÉ, K. M.; REIS, R. P.; PAIVA, F. D. Mercado de carnes Brasil-Rússia: uma análise a partir da perspectiva da nova economia institucional. Organizações Rurais \& Agroindustriais, Lavras, v. 15, n. 1, p.75-86, 2013.

THOMÉ, K. M.; VIEIRA, L. M.; SANTOS, A. C. International marketing channels for Brazilian beef: comparison between Russia and the United
Kingdom. Journal of East-West Business, Essex, v. 18, n. 4, p. 301-320, Dec. 2012.

VIANA, C.; FAGUNDES, M. A internacionalização das indústrias de couro bovino de Mato Grosso do Sul sob a ótica do modelo de Kraus. Revista de Economia \& Relações Internacionais, São Paulo, v. 12, v. 22, p. 136-142, jan. 2013.

VILPOUX, O. Desempenho dos arranjos institucionais e minimização dos custos de transação: transações entre produtores e fecularias de mandioca. Revista de Economia e Sociologia Rural, Brasília, v. 49, n. 2, p. 271-294, abr./jun. 2011.

WACKER, J. G.; YANG, C.; SHEU, C. A transaction cost economics model for estimating performance effectiveness of relational and contractual governance: theory and statistical results. International Journal of Operations \& Production Management, United Kingdom, v. 36, n. 11, p. 1551-1575, May 2016.

WILLIAMSON, O. E. Examining economic organization through the lens of contract. Industrial and Corporate Change, Oxford, v. 12, n. 4, p. 917-942, Aug. 2003.

WILLIAMSON, O. Transaction cost economics and organization theory. Journal of Industrial and Corporate Change, Oxford, v. 2, n. 1, p. 107-156, 1993.

WILLIAMSON, O. E. The economic institutions of capitalism. New York: The Free Press, 1985. 450 p.

WILLIAMSON, O. Markets and hierarchies. New York: Free Press, 1975. 286 p.

The economic institutions of capitalism. New York: The Free Press, 1985. 450 p.

. Markets and hierarchies: analysis and antitrust implications. New York: Free Press, 1975. 286 p.

. Transaction cost economics and organization theory. Journal of Industrial and Corporate Change, Oxford, v. 2, n. 1, p. 107-156, 1993. 